\title{
RELACIÓN ENTRE LOS NIVELES DE ÁCIDO TODO TRANS-REIINOICO EN SANGRE DE CORDÓN UMBILICAL Y MEDIDAS ANTROPOMÉIRICAS DEL RECIÉN NACIDO
}

\author{
Victoria Espinosa F. PhD 1,2,a, Vanesa Boldoy S.1,b, Alejandro Villalobos F.1,b, Silvia \\ Sepúlveda B. PhD ${ }^{1, c}$ \\ ${ }^{1}$ Facultad de Ciencias Médicas, Escuela de Medicina, Universidad de Santiago de Chile. ${ }^{2}$ Laboratorio de Investigación \\ Científica Emory Black.
}

aBioquímica, '⿳Matrón/a, 'Ingeniera Ejecución Química.

\section{RESUMEN}

Antecedentes: La vitamina A, principalmente en su forma de ácido todo trans-retinoico, desempeña una función de vital importancia durante el desarrollo gestacional en todos los tejidos y órganos del cuerpo. Objetivo: Determinar los niveles de ácido retinoico en el suero de sangre de cordón umbilical y relacionarlos con el peso/edad gestacional del recién nacido y el consumo materno de vitamina $A$, conjuntamente con variables socio-económicas y nutricionales. Método: Estudio descriptivo de corte transversal, analizando un total de 62 neonatos y sus madres, provenientes de las maternidades de los Hospitales San José y Barros Luco-Trudeau, Santiago de Chile. Resultados: En la población en estudio se observó una relación directa entre el peso/edad gestacional del neonato y el consumo materno de vitamina A, pero no con los niveles de esa vitamina en el suero de sangre de cordón umbilical. Se encontró que el consumo medio de vitamina A en las embarazadas fue sólo de $2298 \pm 1416,8 \mathrm{UI}$, siendo la dosis recomendada por OMS de $8000 \mathrm{UI}$. Conclusión: Existe asociación entre la ingesta de vitamina A y el estado nutricional materno en relación con el peso/edad gestacional del neonato. Debido a que los requerimientos nutricionales del feto están privilegiados por sobre los maternos, no se observaron diferencias en las características antropométricas del neonato asociadas a la ingesta materna de vitamina $A$.

\section{PALABRAS CLAVES: Vitamina A, ácido trans-retinoico, nutrición materna, nutrición fetal}

\section{SUMMARY}

Background: Vitamin A, mainly in their form of retinoic acid, it carries out a function of vital importance during the gestational development, in all the tissues and organs of the body. Objective: To evaluate the levels of retinoic acid in the serum of blood from umbilical cord and to relate them with the weight/gestational age ratio of the newborns and the maternal intake of vitamin $A$, jointly with socioeconomic and nutritional variables. Method: A descriptive study of traverse court, in the maternities of the Hospitals San José and Barros Luco-Trudeau of Santiago, Chile, studying a total of 62 cases. Results: In the population in study, a direct relationship was observed between the newborn ratio weight/gestational age and the maternal consumption of vitamin A, but no relationship was found among the same weight/gestational age ratio and the levels of this vitamin in the serum of blood of umbilical cord. On the other hand, it was found that the 
average consumption of vitamin A in the maternities it was of $2298 \pm 1416.8 \mathrm{IU}$, being the dose recommended by WHO for pregnant of $8000 \mathrm{IU}$, therefore, the whole population in study had a faulty consumption of vitamin A. Conclusion: Exists association among the intake of vitamin A and the maternal nutritional state with the weight/gestational age ratio of the newborn and the concentration of trans-retinoic acid in the umbilical cord. Because the nutritional requirements of the fetus are privileged over the maternal ones, differences were not observed in the newborn anthropometrics features associated to the maternal vitamin A intake.

\section{KEY WORDS: Vitamin A, trans-retinoic acid, maternal nutrition, fetal nutrition}

\section{INTRODUCCIÓN}

Vitamina A es un término genérico para designar cualquier compuesto que posea las propiedades biológicas del retinol. La vitamina A ejerce sus funciones a través de los metabolitos oxidados del retinol, entre ellos el todo trans-retinal, involucrado principalmente en el ciclo visual de la mayoría de los animales, y el ácido todo trans-retinoico (ATRA), la principal forma activa involucrada en la trascripción de DNA, que actúa como una hormona soluble en lípidos, regulando la proliferación, crecimiento y diferenciación celular y por lo tanto, esencial para el desarrollo gestacional (1-3).

La vitamina $A$, unida a proteínas específicas es transferida de la madre al feto a través de la placenta mediante difusión unidireccional (4-6). Se ha descrito la aparición de anormalidades obstétricas como malformaciones fetales, retardo del crecimiento intrauterino (RCIU) y abortos espontáneos durante la gestación de animales sujetos a hipovitaminosis A $(7,8)$ o hipervitaminosis A $(9)$. En embarazadas también se han descrito patologías como RCIU y abruptos placentarios asociados con concentraciones anormales de retinol sérico (10). La hipovitaminosis A, a largo plazo, afecta el sistema reproductivo femenino y masculino, dando como resultado esterilidad reversible $(4,5)$. Por otra parte, los niños con bajas concentraciones de vitamina $A$ al nacimiento, tienen mayor riesgo de morbilidad, asociado al debilitamiento de su sistema inmunitario (11).

Una hipervitaminosis A aguda durante el período embriogénico del embarazo humano puede producir efectos teratológicos (11), por lo que la OMS recomienda una ingesta diaria media de 8000 Unidades Internacionales (UI) durante el período de gestación. También se han utilizado suplementos diarios de $10.000 \mathrm{UI}$ para prevenir las anomalías fetales provocadas por la deficiencia de vitamina $A$, aunque sólo en ciertas regiones de África y Asia donde esto es frecuente (12).

Existen pocos estudios en relación con los niveles de vitamina $A$ en embarazos patológicos en mujeres mal nutridas y menos aún en mujeres con nutrición y embarazos normales $(11,13)$. Por lo tanto, el objetivo de esta investigación fue determinar si existe una relación entre el estado de nutrición materna, los niveles de vitamina $A$ en suero de sangre de cordón umbilical y la relación peso/edad gestacional del recién nacido.

\section{PACIENTES Y MÉTODOS}

Se determinaron los niveles de vitamina $A$ mediante cromatografía líquida de alta eficiencia (CLAE), y los resultados se asociaron con medidas antropométricas del recién nacido. Esta información fue apoyada con la confección de un perfil bio-socioeconómico de la embarazada y sus niveles de ingesta de vitamina $A$, utilizando los datos obtenidos de la ficha clínica conjuntamente con una encuesta personal.

Estudio descriptivo de corte transversal, realizado en las maternidades de los Hospitales San José y Barros Luco Trudeau, los cuales son representativos de las áreas norte y sur de la Región Metropolitana de Santiago de Chile. Se incorporaron al estudio las mujeres cuyo parto tuvo lugar en las maternidades mencionadas $(n=62)$, cursando embarazos de término (37 a 41 semanas de gestación por fecha de última menstruación segura y/ o edad gestacional determinada por ecografía precoz $<12$ semanas), sin enfermedades previas, con feto único, sin historia de infección o fiebre en los últimos 7 días y con un mínimo de ocho controles de embarazo.

Se obtuvo información de antecedentes maternos obstétricos, familiares, personales y características sociodemográficas. Con el fin de evaluar las diferencias a nivel socioeconómico y cultural entre ambas poblaciones, también se solicitó información de escolaridad, ocupación, paridad, edad de la madre e ingesta de alimentos seleccionados a través de una encuesta nutricional. A los datos descritos se sumaron los antecedentes antropométricos y de morbilidad del recién nacido.

Las variables maternas consideradas fueron: 
edad, paridad, ocupación, nivel educacional, tipo de parto, clasificación de acuerdo a nivel nutricional, historial de enfermedades asociadas con el embarazo y nivel de ingesta de vitamina A. Por otra parte las variables del recién nacido consideradas en el estudio incluyeron: niveles de ATRA de sangre de cordón umbilical, clasificación de relación peso/talla para la edad gestacional.

Para el análisis de las variables, éstas fueron clasificadas de acuerdo con su nivel de medición y tamaño de la muestra. Se determinaron medidas de tendencia central y se utilizaron las pruebas de ANOVA, correlación de Pearson, Rho de Sperman y tau_b de Kendall con un nivel de significancia de $\mathrm{p} \leq 0,05$.

Preparación de la muestra: La sangre de cordón umbilical (aproximadamente $10 \mathrm{ml}$ ) se recolectó desde el lado placentario, inmediatamente después de producido el corte del cordón, utilizando tubos de $15 \mathrm{ml}$ conteniendo $2 \mathrm{ml}$ de citrato de sodio como anticoagulante. Las muestras sanguíneas fueron transportadas refrigeradas y protegidas de la luz hasta el laboratorio, para ser analizadas en un plazo no superior a las 3 horas. Se centrifugaron por 10 minutos a $2500 \mathrm{rpm}$ y a $4^{\circ} \mathrm{C}$, el suero obtenido fue mantenido a $4^{\circ} \mathrm{C}$ para su posterior análisis.

Extracción de la fase lipídica: A $600 \mu \mathrm{L}$ de suero, se agregó igual volumen de diclorometano, se agitó 20 segundos y se centrifugó 2 min a $12500 \mathrm{rpm}$. La fase orgánica fue separada y el solvente evaporado por 30 min a presión reducida a $37^{\circ} \mathrm{C}$ bajo atmósfera de N2. Se obtuvo un residuo lipídico conteniendo la vitamina $A$.

Análisis por HPLC: $1 \mathrm{mg}$ del residuo fue suspendido en $100 \mathrm{~mL}$ de una mezcla 9:1 de acetonitrilo: agua y centrifugado por 30 segundos a 12500 rpm. Un volumen de $20 \mathrm{~mL}$ del sobrenadante, fue cromatografiado en una columna $(1,6 \times 25 \mathrm{~cm})$ de fase reversa $\mathrm{C} 18$ (Lichrosorp), eluyendo con una mezcla 9:1 de acetonitrilo: agua, a $1 \mathrm{~mL} / \mathrm{min}$, con detección a $350 \mathrm{~nm}$ y una presión de 1200 psi, durante 25 min. Se utilizó un cromatógrafo líquido de alta eficiencia Merck-Hitachi, modelo L - 6200; con detector UV-VIS Merck-Hitachi, modelo L - 4250 $(14,15)$. Acetonitrilo y diclorometano, grado HPLC y ácido todo trans-retinoico y citrato de sodio, adquiridos a Sigma-Aldrich, St Louis, MO.

Los niveles de ATRA fueron determinados en base a una curva de calibración concentración/ área, utilizando como estándar una muestra de origen comercial. Los valores de vitamina A obtenidos de sangre de cordón umbilical se clasificaron en intervalos según la propuesta de la OMS (16). Para el diagnóstico de hipovitaminosis A se consideró como punto de corte $<1,05 \mathrm{mmol} / \mathrm{L} \quad(<30$ $\mathrm{mg} / \mathrm{dl}$ ) de acuerdo con lo sugerido por diversos autores (17-19).

\section{RESULTADOS}

Las características principales de la población de las madres en estudio se resumen en la Tabla I. Ambas poblaciones no presentan diferencias estadísticamente significativas respecto de su edad promedio, paridad, ocupación, nivel de escolaridad, estado civil ni en el porcentaje de presentación de patologías asociadas al embarazo. En general, participaron mujeres de más de 30 años, multíparas, dueñas de casa, con escolaridad media completa, casadas y sin patologías asociadas al embarazo. El parto ocurrió en un $60 \%$ de la población en estudio entre la semana 39 y 40 de gestación.

La información sobre el nivel de ingesta de vitamina $A$ se obtuvo a través de una encuesta nutricional informada, la que dejó de manifiesto que el promedio de consumo de vitamina $A$ en la población de madres fue ligeramente superior a $2000 \mathrm{UI}$ diarias. Es importante hacer notar que cerca de un $30 \%$ de la población informó un consumo menor a las $2000 \mathrm{UI}$ diarias (Tabla II).

La clasificación nutricional para embarazadas según edad gestacional, determinó que casi el 50\% de la población correspondía a madres obesas y menos del $10 \%$ se encontraba bajo el peso correspondiente a su periodo de gestación (Tabla III).

El estudio de la distribución de la relación peso/ edad gestacional entre los neonatos (Tabla IV), mostró que casi un $36 \%$ de ellos presentaba una relación adecuada para la edad gestacional, a pesar de la baja ingesta de vitamina A que se observó entre las madres. Los pequeños para la edad gestacional se presentaron en igual cantidad dentro del grupo en estudio, la población de neonatos grandes para la edad gestacional no superó

\section{Tabla I}

\section{CARACTERÍSTICAS GENERALES DE LA POBLACIÓN DE MADRES EN ESTUDIO}

\begin{tabular}{lc}
\hline Características maternas & \\
\hline Edad materna (años) & $31,7 \pm 4,3$ \\
Paridad (multípara) & $80,6 \%$ \\
Ocupación (dueña de casa) & $81,4 \%$ \\
Escolaridad (educación media completa) & $66,1 \%$ \\
Estado civil (casada) & $52,5 \%$ \\
Ausencia de patologías durante el embarazo & $77,4 \%$
\end{tabular}


Tabla II

\section{INGESTA DIARIA DE VITAMINA A DE LAS MADRES DEL GRUPO EN ESTUDIO}

\begin{tabular}{lc}
\hline Ingesta de vitamina $A(U I)$ & $n(\%)$ \\
\hline$\leq 2000$ & $18(29,1)$ \\
$2001-3000$ & $15(24,2)$ \\
$3001-4000$ & $11(17,8)$ \\
$4001-5000$ & $13(20,9)$ \\
$5001-6000$ & $2(3,2)$ \\
$\geq 6001$ & $3(4,8)$ \\
\hline Total & $62(100)$ \\
\hline Promedio de ingesta materna & $2.298 \pm 1416,8$
\end{tabular}

Tabla III

DISTRIBUCIÓN DE LAS MADRES DE ACUERDO A SU ESTADO NUTRICIONAL DETERMINADO POR LA RELACIÓN TALLA/PESO, CON LA EDAD GESTACIONAL

\begin{tabular}{lrl}
\hline Estado nutricional materno & $n$ & $(\%)$ \\
\hline Bajo peso & 4 & $(6,5)$ \\
Peso normal & 15 & $(24,2)$ \\
Sobrepeso & 13 & $(20,9)$ \\
Obesas & 30 & $(48,4)$ \\
\hline Total & 62 & $(100)$
\end{tabular}

Tabla IV

\section{DISTRIBUCIÓN DE LA RELACIÓN PESO/EDAD GESTACIONAL ENTRE LOS NEONATOS EN ESTUDIO Y SU} RELACIÓN CON LOS NIVELES DE ATRA EN SANGRE DE CORDÓN UMBILICAL

\begin{tabular}{lcc}
\hline Relación peso/edad gestacional del recién nacido & $n(\%)$ & $\begin{array}{c}\text { Promedio de ATRA }(\mu M) \text { en sangre de } \\
\text { cordón umbilical }\end{array}$ \\
\hline Adecuado para la edad gestacional & $22(35,5)$ & $10,71 \pm 4,29$ \\
Pequeño para la edad gestacional & $22(35,5)$ & $10,11 \pm 8,29$ \\
Grande para la edad gestacional & $18(29,0)$ & $9,29 \pm 3,91$ \\
\hline Total & $62(100)$ & $10,09 \pm 5,97$
\end{tabular}

ATRA: Ácido todo trans-retinoico.

el $30 \%$ de la muestra. Al comparar, por categorías, los valores de media de vitamina $A$ en la sangre de cordón umbilical de los neonatos a través de la prueba t de Student, encontramos que no existe diferencia significativa entre los valores de las medias del nivel de vitamina A en sangre de cordón umbilical entre las categorías tanto pequeño como adecuado para la edad gestacional. De igual manera se encontró que el nivel de vitamina $A$ entre los neonatos adecuados y los grandes para la edad gestacional no presentaron diferencias significativas entre sí. Cuando se analizan los promedios de concentración de ATRA en la sangre de cordón umbilical de los recién nacidos y se relacionó con la clasificación de peso/edad gestacional de los mismos se determinó que no existía diferencia significativa entre los promedios de los grupos de acuerdo con ANOVA ( $p=0,7552)$.

Al relacionar los datos de peso/edad gestacional de los neonatos con la ingesta diaria de vitamina A por parte de las madres y su estado nutricional se observó que no existe diferencia significativa entre los promedios de cada grupo de acuerdo con ANOVA ( $p=0,5696)$ y no hay interacción significativa entre el estado nutricional mater- no y la clasificación del recién nacido de acuerdo a su relación peso/edad gestacional. A pesar que en los tres grupos de recién nacidos (AEG, PEG y GEG), la mayoría de las madres se clasificó como obesas (aproximadamente un $50 \%$ de cada grupo), su nivel de ingesta de vitamina A no alcanzó a presentar diferencia estadísticamente significativa con la de los otros grupos de madres (Tabla V).

Al determinar los niveles de ATRA en la sangre de cordón umbilical de los recién nacidos se pudo determinar que casi el $70 \%$ de la población presentaba una concentración menor a 10,9 $\mu \mathrm{M}$ (Tabla VI).

A través de la prueba estadística de asociación de Pearson, se comprobó, para la población en estudio, la existencia de una relación directa (positiva media) entre el consumo de vitamina A materno y la relación peso/edad gestacional del neonato y el contenido de vitamina $A$ en la sangre de cordón umbilical $(p=0,01)$ y un $99 \%$ de nivel de confianza. Sin embargo, no fue posible encontrar la misma relación al asociar la concentración de vitamina $A$ en el suero de sangre de cordón umbilical con la relación de peso/edad gestacional, lo que invalida nuestra propuesta inicial de la exis- 
Tabla V

RELACIÓN ENTRE LA MEDIDA DE PESO/EDAD GESTACIONAL DEL RECIÉN NACIDO, LOS NIVELES DE INGESTA MATERNOS DE VITAMINA A DIARIAS Y SU ESTADO NUTRICIONAL

\begin{tabular}{llcl}
\hline $\begin{array}{l}\text { Relación peso/edad gestacional } \\
\text { del } R N\end{array}$ & $\begin{array}{l}\text { Estado nutricio- } \\
\text { nal materno }\end{array}$ & $n$ & $\begin{array}{c}\text { Promedio de ingesta materna } \\
\text { diaria de vitamina } A(U I)\end{array}$ \\
\hline Adecuado para la edad gestacional & Bajo peso & 0 & $2659,8 \pm 667,3$ \\
& Normal & 8 & $3777,7 \pm 872,9$ \\
& Sobre peso & 4 & $3778,6 \pm 1081,5$ \\
& Obesa & 10 & $2554,3 \pm 655,8$ \\
\hline & Total & 22 & $2403,0 \pm 1532,4$ \\
Pequeño para la edad gestacional & Bajo peso & 3 & $2201,5 \pm 1393,4$ \\
& Normal & 6 & $1287,5 \pm 245,5$ \\
& Sobre peso & 2 & $2442,5 \pm 900,1$ \\
\hline & Obesa & 11 & $2443,2 \pm 1018,5$ \\
\hline \multirow{2}{*}{ Grande para la edad gestacional } & Total & 22 & \\
& Bajo peso & 0 & $5199,0 \pm 1023,0$ \\
& Normal & 3 & $5010,3 \pm 1222,4$ \\
& Sobre peso & 4 & $3490,3 \pm 1366,0$ \\
\hline
\end{tabular}

tencia de una relación directa entre ambos parámetros.

A través de la prueba de coeficiente y correlación de rangos ordenados de tau_b de Kendall se observó una relación directa entre el estado nutricional materno y el nivel de vitamina $A$ en la sangre de cordón umbilical $(p<0,05)$. De igual manera, se logró determinar la existencia de una relación entre el consumo de vitamina A materno con la medida de peso/edad gestacional $(p<0,01)$.

\section{DISCUSIÓN}

El análisis de las características sociodemográficas del grupo de madres en estudio y las pertenecientes a dos maternidades mostró que ambas presentan similares características socioeconómicas y culturales, y que podrían haber influenciado el tipo de alimentos que ingirieron durante el embarazo y por lo tanto el aporte nutricional que recibió el feto.

Diversos estudios han señalado la importancia de la alimentación materna en el adecuado desarrollo tanto del embrión como del feto y del recién nacido, encontrándose dentro de los factores nutricios indispensables las vitaminas requeridas como cofactores para el normal funcionamiento fisiológico y metabólico (4).
La carencia de vitamina $A$ en la dieta de una embarazada, se ha asociado a una mayor tasa de abortos y de malformaciones embrionarias, así como también a mayores tasas de retardo del crecimiento intrauterino $(7,8)$, en este ultimo caso en concordancia con los resultados de nuestro estudio, considerando que la dosis dietética recomendada por la Organización Mundial de la Salud para embarazadas es de 800 retinol equivalentes (1 retinol equivalente $=10 \mathrm{UI}$ ), encontramos que las participantes en nuestro grupo de estudio tenían un consumo deficiente de vitamina $A$. En zonas con desnutrición endémica se han implementado programas de suplementación alimentaria

Tabla VI

DISTRIBUCIÓN DE LOS NIVELES DE ÁCIDO TODO TRAS-RETINOICO (ATRA) EN LA SANGRE DE CORDÓN UMBILICAL DE LOS RECIÉN NACIDOS EN ESTUDIO

\begin{tabular}{lc}
\hline ATRA $(\mu M)$ & $n(\%)$ \\
\hline$\leq 10,9$ & $43(69,4)$ \\
$11-20,9$ & $16(25,8)$ \\
$21-30,9$ & $2(3,2)$ \\
$\geq 31$ & $1(1,6)$ \\
\hline Total & $62(100)$
\end{tabular}


que incluyen dietas ricas en esta vitamina, pero en Chile no existe dicho plan, aún cuando se ha observado que en la mayoría de los países en vías de desarrollo existe hipovitaminosis A (20).

Nuestro grupo de estudio presenta un perfil socioeconómico de clase media baja, con gestantes obesas, pero malnutridas, cuya dieta según la información entregada por la encuesta nutricional, casi no incluye alimentos ricos en precursores de la vitamina A tales como zanahoria, zapallo y vegetales de hojas verde oscura.

No existen valores de referencia de los niveles de vitamina A que definan los estados subclínicos o de deficiencia bioquímica de la vitamina $A$ en las gestantes y más aún en los recién nacidos. Algunos autores han señalado que una concentración sérica menor a $30 \mu \mathrm{g} / \mathrm{dL}$ podría definirse como deficiente durante el embarazo (21). Adicionalmente, se ha descrito que probablemente la baja ingesta de vitamina A contribuya a la aparición de la anemia durante el embarazo, de alta prevalencia en Sudamérica, ya que esta vitamina contribuye a la absorción y utilización del hierro, factor que no fue determinado en el presente estudio $(22,23)$.

Existe controversia respecto del estatus materno de vitamina $A$ y los niveles de la misma encontrados en la sangre de cordón umbilical $(24,25)$. Algunos autores señalan que existe correlación entre los niveles de vitamina $A$ en la madre y en la sangre de cordón umbilical $(26,27,28)$. Esto último concuerda con los resultados obtenidos en nuestro estudio. De igual manera se ha explicado que los niveles bajos de vitamina $A$ en los recién nacidos, en comparación con sus madres, puede ser atribuida a problemas en la transferencia a través de la placenta (26).

Se han relacionado también los bajos niveles séricos de vitamina $A$ en la sangre de cordón umbilical con nacimientos prematuros $(28,29)$. En nuestro estudio se seleccionó una muestra con un promedio de edad gestacional al momento del parto dentro del rango de término, aún en los casos de madres mal nutridas y con hipovitaminosis A. Por otra parte, la hipovitaminosis A materna se asocia a una mal nutrición y una ingesta deficiente de antioxidantes, esto último produce un incremento del estrés oxidativo, lo que se relaciona con la patofisiologia de la preeclampsia (30). En nuestro estudio, un 19\% de las madres presentó síndrome hipertensivo del embarazo y en ellas los niveles de ingesta de vitamina $A$ se encontraban dentro de los niveles más bajos, en concordancia con lo descrito en la literatura.
Al igual que estudios realizados en poblaciones brasileñas (31) e inglesas (29), que apoyan la antigua propuesta de que, en general, las necesidades nutricias del embrión se sobreponen a las carencias maternas (32), nosotros encontramos una relación directa entre la ingesta materna de vitamina A y la relación peso/edad gestacional.

Sobre la base de nuestros resultados podemos concluir que existe relación entre la ingesta de vitamina $A$ y el estado nutricional materno con la relación peso/edad gestacional del neonato en los grupos estudiados. Por lo que consideramos muy importante controlar los niveles de ingesta de vitamina $A$ en el periodo pre concepcional y durante el embarazo y suplementarlo cuando corresponda.

Agradecimientos: Este estudio fue financiado con el aporte de la Vicerrectoría de Investigación y Desarrollo de la Universidad de Santiago de Chile, a través de proyecto de investigación Básico-Clínica año 2002.

\section{BIBLIOGRAFÍA}

1. Azaïs-Braesco V, Pascal G. Vitamin A in pregnancy: requirements and safety limits. Am J Clin Nutr 2000;71(suppl):1325S-33S.

2. Blomhof RB, Green JB, Berg T, Norum KR. Vitamin A metabolism: new perspectives on absorption, transport and storage. Physiol Rev 1991;71:951-90.

3. Underwood BA. Vitamin A in human nutricion: public health. In: Sporn MB, Roberts AB, Goodman DS, eds. The retinoids: biology, chemistry and medicine. 2nd ed. New York: Raven Press, 1994:211-29.

4. Rosso P, Mardones F. Nutrición en el embarazo. En: Obstetricia, Pérez Sánchez. A. Pérez, E. Donoso (eds). 3aㅡ Edición. Santiago, Chile: Mediterráneo, 1999; 233-44.

5. Neela J, Raman L. The relationship between maternal nutritional status and spontaneus abortion. Natl Med J India 1997;10:15-6.

6. Rosa F. International experience with retinoic acid embryopathy. Teratology 1991;43:419-25.

7. Eskild W, Hansson V. Vitamin A functions in the reproductive organs. In: Blomhoff RB, ed. Vitamin A health and disease. New York: Marcel Dekker, 1994:531-59.

8. Van Pelt AMM, Van Dissel-Emiliani FMF, Gaemers IC, Van den Burg M, Tanke HJ, De Rooij DG. Characteristics of aspermatogonia and preleptotene spermatocytes in the vitamin A deficient rats testis. Biol Reprod 1995;53:570-8.

9. Morris-Kay G, Sokolova N. Embryonic development and pattern formation. FASEB J 1996;10:961-8.

10. Shah RS, Rajalakshmi R. Vitamin A status of the newborn in relation to gestational age, body weight, 
and maternal nutritional status. Am J Clin Nutr 1984;40:794-800.

11. Sapin V, Alexandre MC, Chib S, Bournazeau JA, Sauvant $P$, Borel $P$, Jacquetin $B$, Grolier $P$, Lèmery D, Dastugue B, Azais-Braesco V. Effect of vitamin A status at the end of term pregnancy on the saturation of retinol binding protein with retinol. Am J Clin Nutr 2000;71(2):537-74.

12. Ziegler EE, Filer LJ,Jr. Conocimientos actuales sobre nutrición. Instituto Internacional de Ciencias de la Vida. $7^{\underline{a}}$ ed. Washington DC: Organización Panamericana de la Salud, 1998, Cap. XI, 118-26.

13. Accioly E, De Souza S. Deficiencia de vitamina A en embarazadas atendidas en una maternidad pública en Río de Janeiro, Brazil. Rev Chil Nutr 2000;27(3): 352-7.

14. Olmedilla B, Granado F, Gil-Martinez F, Blanco I, Rojas-Hidalgo E. Reference values for retinol, tocopherol, and main carotenoids in serum of control and insulin-dependent diabetic Spanish subjects. Clim Chem 1997;43:1066-71.

15. Gamble M, Ramakrishnan R, Palafox N, Briand K, Berglund L, Blaner W. Retinol binding protein as a surrogate measure for serum retinol: studies in vitamin A deficient children from the Republic of the Marshall Islands. Am J Clin Nutr 2001;73(3):594-601.

16. WHO (World Health Organization) Indicators for assessing vitamin A deficiency and their application in monitorin and evaluating intervention programmes, Geneva, WHO/Nut/96-10, 1996; 66.

17. Ortega RM, Andrés $P$, Martinez RM, López-Sovaler AM. Vitamin A status during the third trimester of pregnancy in Spanish women: Influence on concentrations of vitamin A in breast milk. Am J Clin Nutr 1997;66: 564-8.

18. Van den Berg H. Vitamin A intake and status. Eur J Clin Nutr 1996;50 Suppl3: S7-12.

19. Ackurt F, Wetherilt H, Loker M, Hacibekiroglu M. Biochemical outcome of pregnancy. Eur $\mathrm{J}$ Clin Nutr 1995;49:613-22.

20. Ramlho R. Deficiencia marginal de vitamina $A$ en recién nacidos, puérperas y pre-escolares atendidos en tres servicios de salud del Municipio de Río de Janeiro. Río de Janeiro: Escuela Nacional de Salud Pública, 1998; pp 76.
21. Radica MS, Bhaskarama P, Balakrishnaa N, et al. Effects of vitamin A deficiency during pregnancy on maternal and child health. Int J Obstet Gynaecol 2002;109:689-93.

22. Hodges RE, Sauberlich HE, Canham JE, et al. Hematopoietic studies in vitamin A deficiency. Am J Clin Nutr 1978;31:876-85.

23. Rush D. Nutrition and maternal mortality in the developing world. Am J Clin Nutr 2000;72:(suppl): 212S-40S.

24. Kiely M, Cogan PF, Kearney PJ, Morrisey PA. Concentration of tocopherols and carotenoids in maternal and cord blood plasma. Eur J Clin Nutr 1999;53:711-5.

25. Yeum KJ, Ferland G, Patry J, Russell RM. Relationship of plasma carotenoids, retinol and tocopherols in mothers and newborn infants. J Am Coll Nutr 1998;17:442-7.

26. Baydas G, Karatas F, Gursu MF, et al. Antioxidant vitamin levels in term and preterm infants and their relation to maternal vitamin status. Arch Med Res 2002;33(3):276-80.

27. Di Mascio P, Murphy ME, Sies H. Antioxidant defense systems: the role of carotenoids, tocopherols and thiols. Am J Clin Nutr 1991;53(Suppl 1):194S200 S.

28. Kiely M, Cogan PF, Kearney PJ, Morrisey PA. Concentrations of tocopherols and carotenoids in maternal and cord blood plasma. Europ J Clin Nutr 1999;53:711-5.

29. Ghebremeskel K, Burns L, Burden TJ, et al. Vitamin $A$ and related essential nutrients in cord blood: relationships with anthropometric measurements at birth. Early Hum Dev 1994;39:177-88.

30. Palan PR, Mikhail MS, Romney SL. Placental and serum levels of carotenoids in preeclampsia. Obstet Gynecol 2001;98:459-62.

31. Rondó PHC, Abbott R, Tomkins AM. Vitamin A and neonatal anthropometry. J Trop Pediatr 2001;47:30710.

32. Venkatachalam PS, Belavady B, Gopalan C. Studies on the vitamin A nutritional status of mothers and infants in poor communities of India. Trop Pediatr 1962;61:262-8. 